\title{
Proposição de um framework da contribuiç̧ão da aprendizagem interorganizacional para as micro e pequenas empresas
}

\author{
Daniela Siqueira Colet Mestre em Administração. Universidade de Passo Fundo (UnPF) - Brasil. danicolet@hotmail.com \\ Anelise Rebelato Mozzato Doutora em Administração. Universidade de Passo Fundo (UnPF) - Brasil. anerebe@upf.com.br
}

\section{RESUMO}

As relações de cooperação estabelecidas entre os diferentes agentes em aglomerações produtivas que envolvem micro e pequenas empresas (MPEs) resultam em interação e aprendizagem interorganizacional (AIO), o quarto nível da aprendizagem, após a individual, grupal e organizacional. Tais relações apresentam-se como estratégia para o desenvolvimento de competências que minimizam a exposição das empresas às incertezas impostas pelo ambiente, viabilizando o atendimento de uma série de necessidades que não poderiam ser supridas individualmente. Nesse sentido, o objetivo deste ensaio teórico consiste em propor um framework que facilite a compreensão das contribuições da AIO para as MPEs que mantém relações de cooperação interorganizacional. Na proposição do framework, fica evidenciado que por meio das práticas do cotidiano e interações ocorridas entre diferentes agentes em espaços sociais de aprendizagem, tanto estruturados (formais) como não estruturados (informais), ocorrerá o compartilhamento de conhecimento, que possibilita a aquisição e a criação de novos conhecimentos que propiciam a ocorrência de episódios de aprendizagem. Assim, ocorre o processo da AIO, o qual pode resultar em inovação e, por consequência, em vantagem competitiva. Portanto, a AIO pode auxiliar as MPEs com novas práticas de gestão, acesso a novas tecnologias, novos conhecimentos e desenvolvimento de novos produtos, serviços e processos. Por fim, salienta-se que o framework pode ser aplicado em diferentes tipos de aglomerações produtivas, auxiliando no entendimento da AIO em MPEs que mantém relações de cooperação interorganizacional, trazendo contribuição teórica para os estudos sobre $\mathrm{AlO}$ e relações interorganizacionais.

Palavras-chave: Aprendizagem Interorganizacional. Cooperação. Relações Interorganizacionais. Micro e Pequenas Empresas.

\section{Proposal for a framework of the contribution of interorganizational learning for micro and small enterprises}

\begin{abstract}
The cooperative relations established between the different agents in productive agglomerations involving micro and small enterprises (SMEsMPEs) result in interorganizational interaction and learning (IOL), the fourth level of learning, after individual, group and organizational learning. These relationships are presented as a strategy for the development of skills that minimize the exposure of companies to the uncertainties imposed by the environment, making it possible to meet a series of needs that could not be met individually. In this sense, the objective of this theoretical essay is to propose a framework that facilitates the understanding of the contributions of the IOL to the MSPEs that maintain relations of interorganizational cooperation. In the proposal of the framework, it is evidenced that through daily practices and through the interactions that occur between different agents in social spaces of learning, in both structured (formal) and unstructured (informal) spaces, knowledge sharing will occur. This process allows the acquisition and creation of new knowledge that enables the occurrence of learning episodes. Thus, the IOL process occurs, and it can result in innovation and, consequently, in competitive advantage. Therefore, IOL can assist SMPEs with new management practices, access to new technologies, new knowledge and development of new products, services and processes. Finally, it should be pointed out that this framework can be applied in different types of productive agglomerations, helping in the understanding of IOL in MPEs that maintain relations of interorganizational cooperation, bringing theoretical contribution to the studies on IOL and interorganizational relations.

Keywords: Interorganizational Learning. Cooperation. Interorganizational Relationships. Micro and Small Business.
\end{abstract}




\section{INTRODUÇÃO}

O contexto organizacional contemporâneo, caracterizado por contínuas mudanças e pelo acirramento da competição, tem movido as organizações a adotarem uma nova postura de atuação, caracterizada pelo constante envolvimento com demais organizações para atender as exigências de mercado e para gerar vantagem competitiva nos negócios. Assim, a cooperação em relações interorganizacionais viabiliza o atendimento de uma série de necessidades das empresas que não poderiam ser supridas individualmente.

A cooperação tem sido estudada intensamente nos últimos tempos, tanto na literatura internacional (HARDY; PHILLIPS; LAWRENCE, 2003; BRITO; MARIOTTO, 2013; CAPDEVILA, 2014), como nacional (TEIXEIRA; TEIXEIRA, 2011; WEGNER; PADULA, 2012; BORTOLASO; VERSCHOORE; ANTUNES JUNIOR, 2013; NESPOLO et al. 2014; SOUZA et al. 2015; VERSCHOORE; WEGNER; BALESTRIN, 2015; WEGNER; BEGNIS; ALIEVI; MAEHLER, 2016) como importante estratégia, indutora de inovação (BOUNCKEN; PESCH; KRAUS, 2015), que contribui com novas oportunidades de negócios, principalmente para as micro e pequenas empesas (MPEs).

Nesse sentido, a cooperação apresenta-se como alternativa vantajosa para as MPEs, principalmente em diferentes aglomerações produtivas (MOZZATO; BITENCOURT, 2014, 2015) que ao consolidar as práticas colaborativas, conquistam força e eficiência por meio da transferência de conhecimento e inovação (BRITO, 2004), promovendo o crescimento e a competitividade, bem como o desenvolvimento social.

As relações de cooperação estabelecidas entre os diferentes agentes nos aglomerados produtivos resultam em interação e aprendizagem interorganizacional (AIO), que tem se apresentado como uma estratégia para o desenvolvimento de competências que minimizam a exposição das empresas às incertezas impostas pelo ambiente, tornando-se uma dimensão representativa no sucesso das organizações (LARSSON et al., 1998; DYER; SINGH, 1998; LANE; LUBATKIN, 1998).

Contudo, o interesse por pesquisas sobre AIO no Brasil pode ser considerado recente, como pode ser observado nos estudos desenvolvidos por Greve (2005), Estivalete, Pedrozo e Begnis (2006), Pardini, Santos e Gonçalves (2008), Balestrin, Verschoore e Reyes Junior (2010), Gollo et al. (2011), Mozzato e Bitencourt (2013), Larentis et al. (2014), Mozzato e Bitencourt (2014), Mozzato, Bitencourt e Grzybovski (2015). Além disso, a AIO é vista como um campo de análise emergente e ainda em formação (ESTIVALETE; PEDROZO; CRUZ, 2008; MOZZATO; BITENCOURT, 2014; MOZZATO; BITENCOURT; GRZYBOVSKI, 2015) que deve fazer parte da agenda das pesquisas futuras, auxiliando assim no avanço do campo de conhecimento (ANTONELLO; GODOY, 2011).

Diante deste contexto, o objetivo deste ensaio teórico consiste em propor um framework que facilite a compreensão das contribuições da AIO para as MPEs empresas que mantém relações de cooperação interorganizacional. Acredita-se que a pertinência desse diálogo está pautada na continuidade do debate sobre o nível interorganizacional de aprendizagem, nível ainda pouco explorado nos estudos da Administração, contribuindo para o melhor entendimento da AIO e a sua importância como estratégia em diferentes configurações interorganizacionais, principalmente para as MPEs que dessas fazem parte.

A seguir, apresenta-se a abordagem teórica que contempla relações interorganizacionais colaborativas: aglomerados produtivos e suas interfaces e a AIO. Logo após, é proposto um framework de análise para melhor compreensão da importância da AIO entre as MPEs que estabelecem relacionamentos interorganizacionais. Por fim, apresentam-se as considerações finais desse ensaio teórico, trazendo sugestões para pesquisas futuras.

\section{REFERENCIAL TEÓRICO}

Neste capítulo são apresentadas as abordagens teóricas que dão suporte para a pesquisa e direcionam para explicar e melhor compreender a temática que este estudo abarca. Ou seja, aqui está a fundamentação teórica utilizada para realização desta pesquisa. 


\subsection{Relações interorganizacionais colaborativas}

As relações interorganizacionais podem ser compreendidas como as transações, os fluxos e as ligações relativamente duradouras que ocorrem entre uma organização e uma ou mais organizações no seu ambiente (OLIVER, 1990). Nesse sentido, rompendo com princípios da estrutura burocrática tradicional, as relações interorganizacionais privilegiam a cooperação, sem, no entanto, eliminar a competição e conflitos existentes em relacionamentos (MOZZATO; BITENCOURT, 2014). Nas diversas formas de relações interorganizacionais, as empresas podem cooperar para competir, no entanto, destaca-se a necessidade da busca de equilíbrio entre a cooperação e a concorrência, o que influencia fortemente nas estratégias empresariais (JORDE; TEECE, 1989).

Os relacionamentos interorganizacionais cooperativos, segundo Larentis et al. (2014, pressupõem interações interpessoais e intergrupais, e estão baseados fundamentalmente na confiança, no comprometimento e na cooperação, na qual os indivíduos aprendem a partir de suas práticas e interações.

Dentre as diferentes formas de relações interorganizacionais encontram-se os arranjos colaborativos interorganizacionais ou aglomerações produtivas que podem ser constituídas de distritos industriais, arranjos produtivos locais, clusters e sistemas locais de produção (REDESIST, 2003). Tais aglomerações produtivas constituem-se em um tipo particular de território, caracterizado por um conjunto de empresas de um mesmo setor de atividades, com uma atuação condicionada por fatores associados à proximidade física e às condições sociais, culturais e institucionais presentes no local (VALE, 2007).

Os aglomerados produtivos colaborativos que mantêm diferentes relações interorganizacionais possuem alguns diferenciais em razão da diversidade de recursos, a participação de agentes econômicos, políticos e sociais e instituições públicas e privadas, interferindo diretamente nos processos inovativos (MAIA; ALVES; ARAÚJO, 2011) e que oferecem suporte e auxílio ao aglomerado produtivo. A viabilidade das diferentes aglomerações produtivas é uma realidade comprovada por meio de pesquisas teóricas e empíricas, nas quais muitas empresas sobrevivem e/ou conquistam mercados ao trabalharem de maneira colaborativa (MOZZATO; BITENCOURT; GRZYBOVSKI, 2015).

Uma relação de cooperação interorganizacional segundo Abbade (2010), acontece quando organizações optam por agir juntas, em prol de um objetivo maior, unindo suas competências e, com isso, aumentando a eficiência organizacional, proporcionando ganhos aos parceiros envolvidos. Corroborando com esta perspectiva, Balestrin e Verschoore (2008) argumentam que a cooperação é fundamental para as organizações, uma vez que possibilita a obtenção de diferenciais competitivos e, resultados econômicos e sociais por meio de ações coletivas e coordenadas.

Muitas são as dificuldades encontradas pelas MPEs, e novas formas de estruturação podem auxiliar no atingimento dos objetivos e obter vantagens competitivas tais como: maior escala e poder de mercado; geração de soluções coletivas; redução de custos e riscos; acúmulo de capital social; aprendizagem coletiva e inovação colaborativa (VERSCHOORE, 2004). A participação, principalmente das pequenas empresas em aglomerados produtivos, segundo Teixeira e Teixeira (2011), auxilia-as no enfrentamento das barreiras e dificuldades. Além disso, a formação de aglomerados produtivos tem sido importantes para o desenvolvimento industrial e para o fomento da inovação empresarial. Assim, trabalhar de forma colaborativa tem evidenciado ganhos aos diferentes agentes econômicos envolvidos, culminando na "eficiência coletiva" (VERSCHOORE; WEGNER; BALESTRIN, 2015) por meio das relações de cooperação.

No âmbito deste estudo trabalha-se com a AIO, um dos benefícios das relações interorganizacionais nas relações entre MPEs, que podem se dar por meio de aglomerado produtivos, tendo em vista que as organizações que assumem configurações organizacionais diferenciadas buscam também, frequentemente, facilitar a difusão do conhecimento (EASTERBY-SMITH; LYLES; TSANG, 2008; BALESTRIN; VERSCHOORE, 2008; WEGNER; 2011), logo, a possibilidade do compartilhamento do conhecimento e AIO por meio das relações de cooperação. 


\title{
2.2 Aprendizagem Interorganizacional
}

As pesquisas especificas sobre a AIO iniciam, principalmente, no final da década de 1990 (LARSSON et al., 1998). Desde então, muitos pesquisadores apontam para a necessidade de aprofundamento dos estudos referentes à $\mathrm{AIO}$, tendo em vista que apesar do reconhecimento de que as relações colaborativas fazem parte das configurações organizacionais, sua compreensão no que se refere-se a AIO ainda é limitada (LARSSON et al.1998; LANE; LUBATKIN, 1998; DIERKES et al., 2001; KNIGHT, 2002; EASTERBY-SMITH; LYLES, 2003; GREVE, 2005; INKPEN; TSANG, 2007; NOOTEBOON, 2008; MOZZATO; BITENCOURT, 2014; MOZZATO; BITENCOURT; GRZYBOVSKI, 2015).

Por AlO entende-se a aprendizagem no contexto de grupos ou pares de organizações que estão proativamente cooperando (KNIGHT, 2002). Dessa forma, por meio da AIO as organizações aprendem e melhoram a sua base de conhecimento e também aprendem como explorar o novo conhecimento que as torna interdependentes (LUBATKIN; FLORIN; LANE, 2001). Greve (2005, p. 1026) explicita que a AIO:

\begin{abstract}
É uma forma distinta de aprendizado porque aorganização aprende com a experiência dos outros ao invés de suas próprias experiências. Enquanto distintivo na fonte de aprendizagem, a aprendizagem interorganizacional é suportada por processos intraorganizacionais de criação e retenção de conhecimento, e algumas das suas descobertas são idênticas às da pesquisa sobre transferência de conhecimento intraorganizacional.
\end{abstract}

Na mesma linha de análise, Larsson et al. (1998, p. 287) reforçam que "a aprendizagem interorganizacional pode, então, ser vista como uma aquisição coletiva de conhecimento entre um conjunto de organizações", diferenciando-se da aprendizagem organizacional pelo fato de incluir a sinergia de aprendizagem, ou seja, os efeitos das interações não poderiam ocorrer, caso não tivesse existido nenhuma interação entre as organizações.

Para Mozzato, Bitencourt e Grzybovski (2015), o nível interorganizacional refere-se às relações estabelecidas pelas organizações além do limite organizacional. Por sua vez, a cooperação está associada às estratégias relacionais estabelecidas entre os diferentes agentes nos relacionamentos interorganizacionais. Assim, este nível interorganizacional de aprendizagem é entendido de maneira diferente da aprendizagem organizacional, em virtude de incluir o efeito da interação entre organizações, permitindo maior sinergia que promove a aprendizagem e a aquisição coletiva de conhecimento entre um conjunto de organizações.

Larsson et al. (1998, p. 289) referem que:

A aprendizagem interorganizacional pode ser alcançada transferindo conhecimento existente de uma organização para outras organizações, como também criando conhecimento completamente novo através da interação entre as organizações. A transferência e a criação de conhecimento requerem transparência e receptividade simultâneas em algum nível entre as organizações.

Contribuindo com a temática da AIO, Mozzato e Bitencourt (2013) com base em pesquisas e múltiplas fontes que tratam da temática, propõe seis elementos constitutivos da AIO, os quais são evidenciados no Quadro 1.

Quadro 1 - Elementos constitutivos da AIO

\begin{tabular}{|l|l|}
\hline $\begin{array}{l}\text { Confiança } \\
\text { estabelecida entre } \\
\text { agentes }\end{array}$ & $\begin{array}{l}\text { Consiste no compromisso mútuo estabelecido entre os diferentes agentes, } \\
\text { refletindo a convicção de que há transparência nos relacionamentos e que a } \\
\text { "verdade" será prezada, assegurando, por consequência, que as obrigações do } \\
\text { relacionamento serão cumpridas. }\end{array}$ \\
\hline $\begin{array}{l}\text { Cooperação entre } \\
\text { Agentes }\end{array}$ & $\begin{array}{l}\text { Consiste em ações colaborativas nas relações interorganizacionais, havendo } \\
\text { compromisso recíproco. Contudo, não se nega a coexistência da competição. }\end{array}$ \\
\hline Interações Sociais & Consiste nas relações sociais estabelecidas entre os diferentes agentes. \\
\hline
\end{tabular}




\begin{tabular}{|l|l|}
\hline Interdependência & $\begin{array}{l}\text { Diz respeito à vinculação entre os diferentes agentes, podendo ser tanto } \\
\text { referente aos objetivos, como aos recursos ou complementaridade na realização } \\
\text { das tarefas. }\end{array}$ \\
\hline Proximidade Social & $\begin{array}{l}\text { Diz respeito a maior identidade entre os diferentes agentes, facilitando o } \\
\text { estabelecimento de laços sociais mais fortes, dessa forma, conduzindo a maior } \\
\text { proximidade social. }\end{array}$ \\
\hline $\begin{array}{l}\text { Suscetibilidade ao } \\
\text { Aprendizado }\end{array}$ & $\begin{array}{l}\text { Relaciona-se à possibilidade de aprendizado por parte dos diferentes agentes, } \\
\text { ficando na dependência tanto da predisposição como da capacidade para } \\
\text { aprender. }\end{array}$ \\
\hline
\end{tabular}

Fonte: Adaptado de Mozzato e Bitencourt (2013).

Com base nos seis elementos constitutivos a AIO, Mozzato e Bitencourt (2013) enfatizam que estes estão interligados e em processo dinâmico de interligação, salientando que a confiança e a cooperação têm íntima relação, os quais envolvem os outros quatro elementos constitutivos da AIO (interações sociais, interdependência, proximidade social, suscetibilidade ao aprendizado).

Nessa linha de análise, a AIO ocorre nas relações de cooperação entre diferentes agentes, melhorando e aumentando as bases de conhecimentos de cada envolvido, e ainda acrescentando potencial para criação de vantagem competitiva individual e a uma determinada configuração interorganizacional (MOZZATO; BITENCOURT, 2014).

Os episódios de aprendizagem emergem no todo relacional do dia a dia em diferentes espaços sociais (estruturados ou não estruturados) nas relações de cooperação (MOZZATO; BITENCOURT, 2014). As mesmas não negam que podem existir barreiras em tais relações de cooperação, as quais certamente afetam a AIO. Em consonância, Knight (2002), Knight e Pye (2005), explicam que os episódios de aprendizagem podem ser entendidos com o fluxo das atividades cotidianas que se dão tanto em espaços formais como informais.

Desse modo, os episódios de aprendizagem são ações e interações que ocorrem entre diferentes organizações, podendo ser entre diferentes agentes num aglomerado produtivo, que propiciam acontecimentos e experiências de aprendizagem (eventos de aprendizagem), desencadeando episódios de aprendizagem. Assim, constituem-se como importantes para a análise do processo da AIO (MOZZATO; BITENCOURT, 2014). Nessa linha de análise, a seguir apresenta-se o framework de análise da AIO para estudos em MPEs que estabelecem relacionamentos interorganizacionais.

\section{FRAMEWORK DA CONTRIBUIÇÃO DA APRENDIZAGEM INTERORGANIZACIONAL PARA AS MICRO E PEQUENAS EMPRESAS}

O processo de AlO tem se destacado como um dos temas emergentes para o ambiente no qual as organizações encontram-se inseridas, constituindo-se em um importante instrumento na formulação e no desenvolvimento de estratégias para as empresas que atuam em diferentes aglomerações produtivas (ESTIVALETE, et al. 2009; MOZZATO; BITENCOURT, 2013). A importância da AIO decorre em razão de que aprendizagem não se apresenta somente como pretexto que leva à formação de aglomerações produtivas, mas também como necessária para que ocorra eficácia na cooperação (LARSSON et al., 1998). Dessa forma, por meio das relações interorganizacionais, a aprendizagem e a inovação colaborativa destacam-se como importantes para o futuro competitivo de diferentes aglomerados produtivos, beneficiando, sobretudo, as MPEs (MOZZATO; BITENCOURT, 2014).

Lubatkin, Florin e Lane (2001) argumentam não apenas a aquisição e transferência de conhecimentos nas relações interorganizacionais são importantes, mas também, a criação do novo conhecimento, tendo em vista que além de aprender e melhorar os seus respectivos conhecimentos, devem aprender a aprender junto com os parceiros, aprendendo a explorar novos conhecimentos interdependentes na aglomeração produtiva. Em consonância, Hardy, Phillips e Lawrence (2003) referem que por meio da colaboração interorganizacional, 
não somente se transfere o conhecimento existente entre organizações, mas se facilita a criação de novos conhecimentos.

Na mesma linha de análise, Dyer e Singh (1998) destacam o compartilhamento de conhecimento e, também Jorde e Teece (1989) evidenciam que além de possibilitar o aumento do acesso a novos conhecimentos, essas configurações facilitam a AIO, o acesso às novas tecnologias, o processo de inovação e permitindo a ampliação da capacidade tecnológica. Assim, Child (2001, p. 676) argumenta que as "alianças estratégicas podem promover a aprendizagem tanto para facilitar a transferência de conhecimento como promover a criação de conhecimento com base em competências complementares". Portanto, a AIO consiste no acesso, na aquisição e na assimilação de conhecimentos (GRANT; BADEN-FULLER, 2004).

Além disso, as relações de cooperação têm sido apontadas como uma nova fonte de inovação, pois apresentam ambientes nos quais os conhecimentos podem ser gerados de forma mais eficiente e rápida (NAKANO, 2005), e muitas empresas são movidas a cooperar com seus concorrentes a fim de obterem uma posição de mercado mais favorável (MORRIS; KOÇAK; OZER, 2007). Portanto, a cooperação interorganizacional também desempenha um papel crucial na capacidade de inovação e competitividade (CAPDEVILA, 2014).

A formação de diferentes aglomerações produtivas com relações de cooperação constitui-se em uma alternativa vantajosa para as micros, pequenas e médias empresas (AMATO NETO, 2000; MORRIS; KOÇAK; OZER, 2007; BALESTRIN; VERSCHOORE, 2008), sendo que a cooperação e a competição coexistem também nessas diferentes configurações. Entretanto, a confiança é considerada como fator imprescindível nos processos de relações interorganizacionais de cooperação, mesmo coexistindo com a competição.

Nesse sentido, apresenta-se a Figura 2, a qual expõe esquematicamente o desenvolvimento teórico e tem o propósito de auxiliar e proporcionar uma melhor compreensão, na medida em que procura estabelecer relações na temática que está sendo estudada, tendo como foco a AlO em contexto de relações interorganizacionais entre MPEs.

A proposição do framework (Figura 2) é apresentada para analisar pequenas e médias empresas que mantêm relações interorganizacionais em organizações em rede (aglomerado produtivo de MPEs). Por meio das práticas do cotidiano e interações ocorridas entre diferentes agentes em espaços sociais de aprendizagem, tanto estruturados (formais) como não estruturados (informais), ocorrerá o compartilhamento de conhecimento, havendo assim a aquisições de conhecimento e, criação de novos conhecimentos que propiciam a ocorrência de episódios de aprendizagem. Assim, ocorre o processo de AIO (constituído da cooperação, confiança, interdependência, interações sociais, suscetibilidade ao aprendizado e proximidade social) que pode resultar em inovação que acrescerá vantagem competitiva. 
Figura 2 - Framework de análise de aglomerado produtivo de MPEs

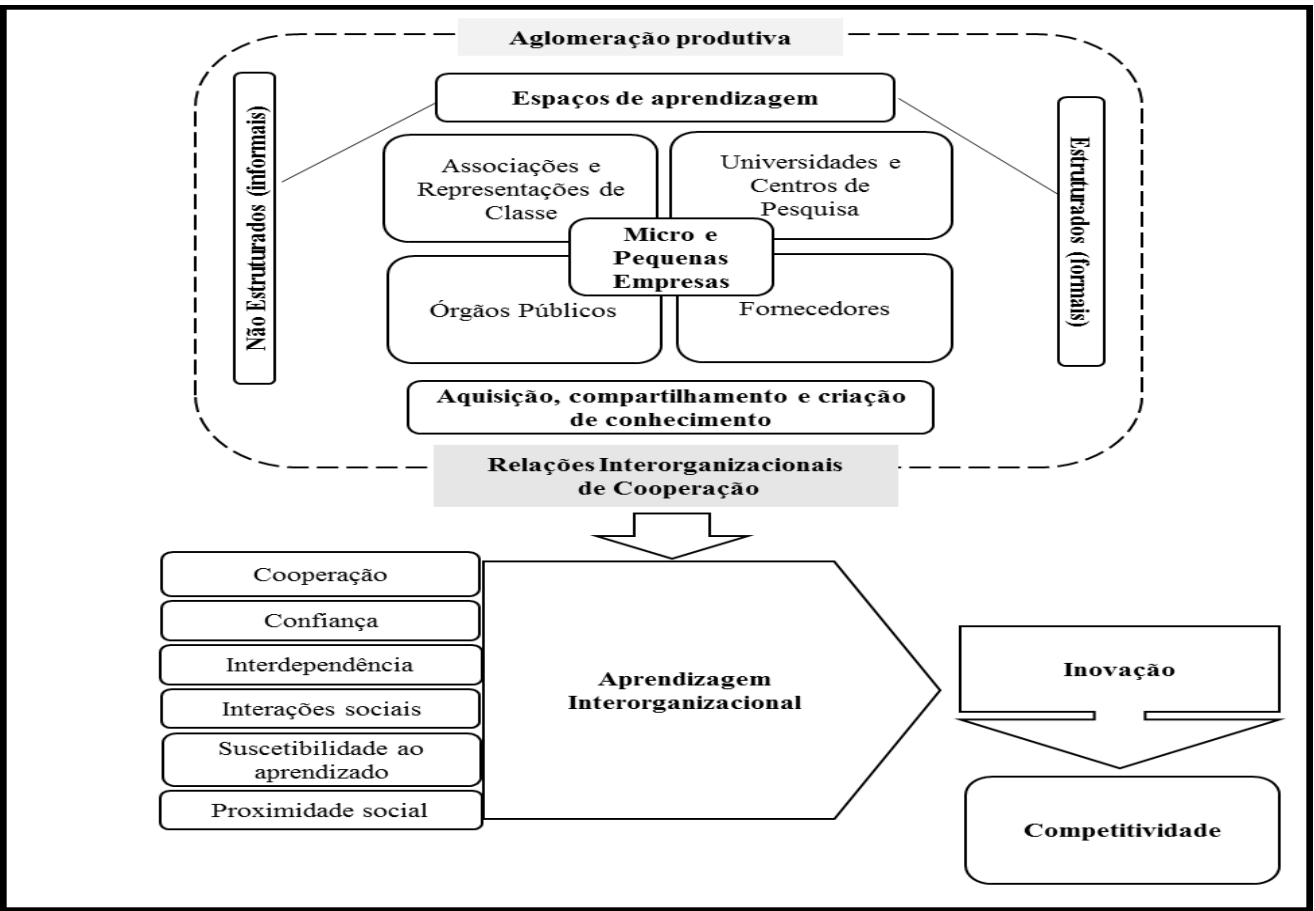

Fonte: Elaborado pelas autoras com base em Dyer e Singh (1998), Jorde e Teece (1989), Larsson et al. (1998), Child (2001), Mozzato e Bitencourt (2014) e Capdevila (2014).

Por meio da Figura 2 acredita-se ficar evidenciada a proposição teórica deste trabalho. Tal figura representa um contexto de MPEs em contexto de aglomerado produtivo, no qual ocorrem as relações interorganizacionais. Os relacionamentos interorganizacionais são facilitados pela cooperação e confiança, possibilitando assim a AIO. Na ocorrência da AlO evidencia-se que a confiança é um pré-requisito da cooperação e a cooperação repercute na confiança. Nesse sentido, por meio das relações interorganizacionais AIO constitui-se como importante tanto para o desenvolvimento de cada MPEs como para as aglomerações produtivas as quais pertencem.

Em vista dos argumentos apresentados, infere-se que a AIO é uma estratégia com potencial de gerar vantagem competitiva para as organizações, sobretudo para as empresas de pequeno porte, em razão de terem recursos financeiros limitados, estrutura, acesso mais dificultado a novas formas de gestão e as novidades do mercado. Por meio da AIO, muitas das dificuldades enfrentadas pelas MPEs podem ser solucionadas, com novas práticas de gestão, acesso a novas tecnologias, novos conhecimentos e inovação, sendo apontada como um dos principais ganhos competitivos das relações interorganizacionais cooperativas por diversos autores, dentre eles: Amato Neto (2000), Mohr e Singupta (2002), Oliveira e Guerrini (2003), Verschoore e Balestrin (2008), Abbade (2010), Klein e Pereira (2013), Fischer et al. (2014).

\section{CONSIDERAÇÕES FINAIS}

As relações de cooperação interorganizacionais facilitam a AIO e a criação de novos conhecimentos, produzindo inovação e novas soluções, principalmente para as MPEs, facilitando o seu espaço no mercado, o que em muitas circunstâncias, não conseguiriam sozinhas. A análise aqui apresentada revela que diferentes configurações organizacionais podem servir como resposta às exigências do mercado e, cooperar constitui-se em uma alternativa estratégica competitiva que vem apresentando expressiva dimensão econômica e social, facilitando e sendo facilitada pela AIO.

Adicionalmente, com a AIO as empresas aprendem com as experiências de outras empresas, e a aprendizagem acontece por meio das relações interorganizacionais que, estão baseadas no comprometimento e na cooperação. Desse modo, a partir das práticas e interações interorganizacionais, 
acresce as bases de conhecimentos de cada envolvido. Assim, a AlO tem se apresentado como uma estratégia para o desenvolvimento de competências que minimizam a exposição das empresas às incertezas impostas pelo ambiente, tornando-se uma dimensão representativa para uma melhor performance organizacional.

Portanto, o framework proposto visando à compreensão das contribuições da AIO, revela a notoriedade da importância da AIO para as MPEs que mantém relações interorganizacionais, inclusive fazendo parte de diferentes aglomerados produtivos, em razão de que muitas das dificuldades enfrentadas podem ser solucionadas, com novas práticas de gestão, acesso a novas tecnologias, criação de novos conhecimentos e inovação. Também, a AIO torna-se necessária para que ocorra eficácia na cooperação, destacando-se como indispensável para o futuro competitivo de diferentes aglomerados produtivos. Dessa forma, verifica-se a sua viabilidade e pertinência. Por fim, não se negam os ajustes que podem ser necessários ao framework, na medida em que as pesquisas com esse propósito avancem.

O framework proposto pode ser aplicado em diferentes tipos de aglomerações produtivas, que auxiliará no entendimento da AIO em MPEs que mantém relações de cooperação interorganizacional. Ele pode ser adaptado para diferentes aglomerações colaborativas, trazendo contribuição teórica para os estudos sobre $\mathrm{AIO}$, que tem sido apontado como tema emergente e relevante, com amplo espaço nas pesquisas acadêmicas. Também demonstra $O$ valor das pesquisas tanto sobre a AlO como das relações de cooperação interorganizacionais, ambas importantes para os estudos organizacionais, ainda mais quando se tem como foco as MPEs.

Do ponto de vista prático, cabe destacar que a aplicação do framework de análise em diferentes configurações interorganizacionais que mantém estratégias de cooperação, pode auxiliar no entendimento e crescimento de tais espaços. Ainda, acredita-se que este estudo fornece subsídios importantes a partir das reflexões aqui propostas, principalmente para as MPEs, evidenciando a importância da AIO em aglomerações produtivas e a relevância de estabelecer relações interorganizacionais como uma alternativa para facilitar o compartilhamento de recursos, de conhecimentos, dentre outros benefícios por meio das relações de cooperação e confiança.

Destaca-se que devido ao fato deste ser um ensaio teórico, não se pretende apresentar conclusões absolutas, mas sim, trazer contribuições para a área de estudo, entendendo que cada vez mais se torna necessário pensar na AIO e em estratégias de cooperação também para as MPEs. Finalmente, sugere-se a realização de outros estudos futuros, tanto teóricos como empíricos, que poderão ser desenvolvidos a partir das evidencias exibidas neste ensaio, ou ainda incorporando novas temáticas, como o estudo das mudanças provocadas pela AIO em uma perspectiva evolutiva e voltada para o desenvolvimento de localidades e melhor qualidade de vida das pessoas envolvidas.

\section{REFERÊNCIAS}

ABBADE, E. B. Cooperação interorganizacional: fonte de aprendizagem e vantagem competitiva ou oportunismo? Revista de Administração da UNIMEP, v. 8, n. 2, p. 154-179, 2010.

AMATO NETO, J. A. Redes de cooperação produtiva e clusters regionais: oportunidades para as pequenas e médias empresas. São Paulo: Atlas, 2000.

ANTONELLO, C. S. Contextos do saber: a aprendizagem informal. In: ANTONELLO, C. S.; GODOY, A. S. (Org.). Aprendizagem organizacional no Brasil. Porto Alegre: Bookman, 2011.

BALESTRIN, A.; VERSCHOORE, J. R.; REYES JUNIOR, E. O campo de estudo sobre redes de cooperação Interorganizacional no Brasil. Revista de Administração Contemporânea, Curitiba, v. 14, n. 3, p. 458-477, mai./jun. 2010.

BALESTRIN, A.; VERSCHOORE, J. Redes de cooperação empresarial: estratégias de gestão na nova economia. Porto Alegre: Bookman, 2008. 
BOUNCKEN, R.B.; PESCH, R.; KRAUS, S.SME innovativeness in buyer-seller alliances: effects of entry timing strategies and inter-organizational learning. Review of Managerial Science, v. 9, n. 2, p. 361-384, 2015.

BRITO, E. P. Z.; MARIOTTO, G. Benefits of Cooperation between Buyers and Providers: a study in the field of information and communications technology. Brazilian Journal of Business Management, v. 15, n. 14, p. 241-261, abr./jun. 2013.

BORTOLASO, I.; VERSCHOORE, J. R.; ANTUNES JUNIOR., J. A. V. Cooperative strategies: evaluating network strategy management of small and medium-sized enterprises. Revista Brasileira de Gestão de Negócios, v. 14, n. 45, p. 419-437, 2012.

BORTOLASO, I. V.; VERSCHOORE, J. R.; ANTUNES JUNIOR., J. A. V. Práticas de gestão de redes de cooperação horizontais: proposição de um modelo de análise. Contabilidade, Gestão e Governança, v. 16, n. 3, p. 3-16, 2013.

CAPDEVILA, I. Different Inter-Organizational Collaboration Approaches in Coworking Spaces in Barcelona. 2014. Disponível em: <https://papers.ssrn.com/sol3/papers.cfm?abstract_id=2502816>. Acesso em: 8 maio 2018. doi.org/10.2139/ssrn.2502816

CHILD, J. Learning through strategic alliances. In: DIERKES, M. et al. (Ed.). Handbook of organizatinal learning \& knowledge. New York: Oxford University: 2001. p. 657-679.

DIERKES, M. et al. Handbook of Organizatinal Learning and Knowledge. Oxford University Press, New York, NY, 2001.

DYER, J.H.; SINGH, H. The relational view: cooperative strategy and sources of interorganizational competitive advantage. Academy of Management Review, v. 23, n. 4, p. 660-679, 1998.

ESTIVALETE, V. F. B.; PEDROZO, E. A.; BEGNIS, H. S. M. Em busca da ação coletiva: estratégias de aprendizagem interorganizacional adotadas pelas organizações que estabelecem relacionamentos horizontais em redes. In: ENCONTRO DA ANPAD, 30., 2006, Salvador. Anais... Salvador: ANPAD, 2006.

ESTIVALETE, V. F. B. et al. Ampliando a compreensão sobre a aprendizagem interorganizacional: um estudo em uma rede do setor de serviços. Gestão \& Regionalidade, v. 25, n. 75, p. 31-44, set./dez. 2009.

ESTIVALETE, V. F. B.; PEDROZO, E. A.; CRUZ, L. B. The learning process in interorganizational elationships. BAR, Curitiba, v. 5, n. 4, p. 319-331, 2008.

EASTERBY-SMITH; LYLES; TSANG. Inter-organizational Knowledge transfer current themes and futures prospects. Journal of Management Studies, v.45, n.4, p.677-690, 2008.

\section{EASTERBY-SMITH, M.; LYLES, M. The blackwell handbook of organizational learning and knowledge} managemet. Oxford: Blackwell Publishing, 2003.

FISCHER, A. et al. Aliança estratégica: rede oeste de cooperação de empresas contábeis de Santa Catarina.

Revista de Contabilidade do Mestrado em Ciências Contábeis da UERJ (online), Rio de Janeiro, v. 19, n. 2, p. 58-78, maio/ago. 2014.

GOLLO, S. S. et al. Rede de cooperação interorganizacional: estudo de caso de uma rede metal-mecânica no Rio Grande do Sul. In: ENCONTRO DE ESTUDOS EM ESTRATÉGIA DA ANPAD, 5., 2011, Porto Alegre.

Anais...Porto Alegre: ANPAD, 2011.

GREVE, H. R. Interorganizational learning and social structure. Organization Studies, v. 26, n. 7, p. 1025-1047, 2005.

GRANT, R. M.; BADEN-FULLER, C. A knowledge accessing theory of strategic alliances. Journal of

Management Studies, v. 41, n.1, p. 61-84, 2004. 
HARDY, C.; PHILLIPS, N.; LAWRENCE, T. B. Resources, knowledge and influence: the organizational effects of interorganizational collaboration. Journal of Management Studies, v. 40, n. 2, p. 321-347, 2003.

INKPEN, A. C; TSANG, E. W. K. Learning and Strategic Alliances. The Academy of Management Annals, v.1, n.1, p. 479- 511, 2007.

JORDE, T. M.; TEECE, D. J. Competition and cooperation: striking the right balance. Business \& Public Policy, v. 31, n. 3, p. 25-37, 1989.

KLEIN, L. L.; PEREIRA, B. A. D. Compreendendo a integração interorganizacional: quais as mudanças que ocorrem na empresa devido a sua entrada em uma rede? Revista Gestão e Planejamento, Salvador, v. 14, n. 3, p. 560-579, set./dez. 2013.

KNIGHT, L. Network learning: exploring learning by interorganizational networks. Human Relations, v. 55, p. 427-454, 2002.

KNIGHT, L.; PYE, A. Network learning: an empirically derived model of learning by groups of organizations. Human Relations, v.58, n.3, p.369-392, 2005.

LANE, P.; LUBATKIN, M. Relative absorptive capacity and interorganizational learning. Strategic Management Journal, v. 19, n. 5, p. 461-477, 1998.

LARENTIS, F.; ANTONELLO, C. S.; MILAN, G. S.; TONI, D. Aprendizagem organizacional e relacionamentos interorganizacionais: um estudo de casos múltiplos. BASE - Revista de Administração e Contabilidade da UNISINOS, v. 11, n. 4, p. 347-366, 2014.

LARSSON, R. et al. The interorganizational learning dilemma: collective knowledge development in strategic alliances. Organization Science, v. 9, n. 3, p. 285-305, 1998.

LUBATKIN, M., FLORIN, J., LANE, P., Learning together and Apart: a model of reciprocal interfirm learning. Human Relations, v. 54, n. 10, p. 1353-1382, 2001.

MAIA, M. H. B.; ALVES, R. R. A.; ARAÚJO, P. G. Cooperação em um Aglomerado Produtivo: O Caso do Setor Moveleiro de Carmo do Cajuru (MG).In: ENCONTRO DA ASSOCIAÇÃ̃O NAICONAL DE PÓS GRADUAÇÃO E PESQUISA EM ADMINISTRAÇÃO, 35., 2011, Rio de Janeiro. Anais... Rio de Janeiro: ANPAD, 2011. p. 1-17.

MORRIS, M. H.; KOÇAK, A.; OZER, A. Coopetition as a small business strategy: implications for performance. Journal of Small Business Strategy, v. 18, n. 1, p. 35-55, 2007.

MOHR, J. SINGUPTA, S. Managing the paradox of interfirm: the role of governance mechanisms. The journal of Business et Industrial Marketing, v. 17, n. 4, p. 282-302, 2002.

MOZZATO, A. R.; BITENCOURT, C. C. Aprendizagem Interorganizacional: Constructo para sua melhor compreensão. In: ENCONTRO EM GESTÃO DE PESSOAS E RELAÇÕES DE TRABALHO DA ANPAD, 4., 2013, Brasília. Anais... Brasília: ANPAD, 2013.

MOZZATO, A. R.; BITENCOURT, C. C. Understanding interorganizational learning based on social spaces and learning episodes. Brazilian Administration Review, v. 11, n. 3, p. 284-301, 2014.

MOZZATO, A. R.; BITENCOURT, C. C.; GRZYBOVISKI, D. The interorganizational level in the learning continuum: analytic conceptual scheme. International Business Research, v. 8, n. 4, p. 94-106, 2015.

NAKANO, D. N. Fluxos de conhecimento em redes interorganizacionais: conceitos e fatores de influência. In: AMARO NETO, J. (Org.) Redes entre organizações: domínio do conhecimento e da eficácia operacional. São Paulo: Atlas, 2005. 
NESPOLO, D. et al. Vantagens competitivas de pequenas e médias empresas com a participação em redes de cooperação: o caso do Mercado Tutto. Revista de Empreendedorismo e Gestão de Pequenas Empresas, v. 3, n. 2, p. 145-160, 2014.

OLIVER, C Determinants of interorganizational relationships: Integration and future directions. Academyof Management Review, v. 15, n.2, p.241-265, 1990.

PARDINI, D. J.; SANTOS, R. V.; GONÇALVEZ, C. A. A dinâmica da aprendizagem intra e interorganizacional: perspectivas em estratégias cooperativas e competitivas utilizando as tipologias de exploration e exploitation. Revista Economia \& Gestão, v. 8, n. 18, p. 134-150, set./dez. 2008.

SOUZA, G. H. S. et al. A Influência das redes de cooperação no desenvolvimento de micro e pequenas empresas (MPEs). Desenvolvimento em Questão, v. 13, n. 31, p. 259-294, 2015.

REDESIST. Glossário sobre arranjos e sistemas produtivos e inovativos locais. 2003. Disponível em: $<$ http://www.redesist.ie.ufri.br>. Acesso em: jul. 2015.

TEIXEIRA, M. C.; TEIXEIRA, R. M. Relacionamento, cooperação e governança em arranjos produtivos locais: o caso do apl de madeira e móveis do estado de Rondônia. Revista Eletrônica de Administração, v. 17, n. 1, p. 237-269, jan./abr. 2011.

VALE, G. M. V. Territórios vitoriosos: o papel das redes organizacionais. Rio de Janeiro: Editora Garamond, 2007.

VERSCHOORE, J. R.; BALESTRIN, A. Ganhos competitivos das empresas em redes de cooperação. Revista de Administração Eletrônica, São Paulo, v. 1, n. 1, p.1-22, jan./jun. 2008.

VERSCHOORE, J. R.; WEGNER, D.; BALESTRIN, A. The evolution of collaborative practices in small-firm networks: a qualitative analysis of four Brazilian cases. Management Practice, v. 8, n. 2, p.152-168, 2015.

VERSCHOORE, J.R.S. Redes de cooperação: concepções teóricas e verificações empíricas. In: VERSCHOORE, J. S. Redes de cooperação: Uma nova organização de pequenas e médias empresas no Rio Grande do Sul. Porto Alegre: FEE, 2004. p. 15-46.

WEGNER, D. Aprendizagem interorganizacional: um estudo das redes horizontais de pequenas empresas. In: ANTONELLO, C. S.; GODOY, A. S. (Org.). Aprendizagem organizacional no Brasil. Porto Alegre: Bookman, 2011. cap. 23.

WEGNER, D.; PADULA, A. D. Quando a cooperação falha: um estudo de caso sobre o fracasso de uma rede interorganizacional. Revista Administração Mackenzie, v. 13, n. 1, p. 145-171, 2012.

WEGNER, D. et al. The dynamics of cooperation: proposal of a life cycle model of small-firm networks. Gestão \& Regionalidade, v. 32, n. 94, p.118-130, 2016.

ZAWISLAK, P. A. From the dream of opportunities to the nirvana of trust: issues for a framework on cooperative agreement stability. Revista Eletrônica de Administração, v. 10, n. 6, p.2-22, 2004. 